Supporting Information for

\title{
Acridine Orange Encapsulated Mesoporous Manganese Dioxide Nanoparticles to Enhance Radiotherapy
}

Junzhi Liu ${ }^{1, \dagger}$, Weizhong Zhang ${ }^{2, \dagger}$, Anil Kumar ${ }^{2}$, Xiaoli Rong ${ }^{3}$, Wei Yang ${ }^{2}$, Hongmin Chen ${ }^{4}$, Jin $X i e^{2, *}$ Yimin Wang ${ }^{3, *}$

${ }^{1}$ Departments of Quality Control, China-Japan Union Hospital of Jilin University, Changchun, Jilin 130033, China

${ }^{2}$ Department of Chemistry, University of Georgia, Athens, Georgia 30602, USA

3 The Scientific Research Center, China-Japan Union Hospital of Jilin University, Changchun, Jilin 130033, China

${ }^{4}$ State Key Laboratory of Molecular Vaccinology and Center for Molecular Imaging and Translational Medicine, School of Public Health, Xiamen University, Fujian 361102,

China 


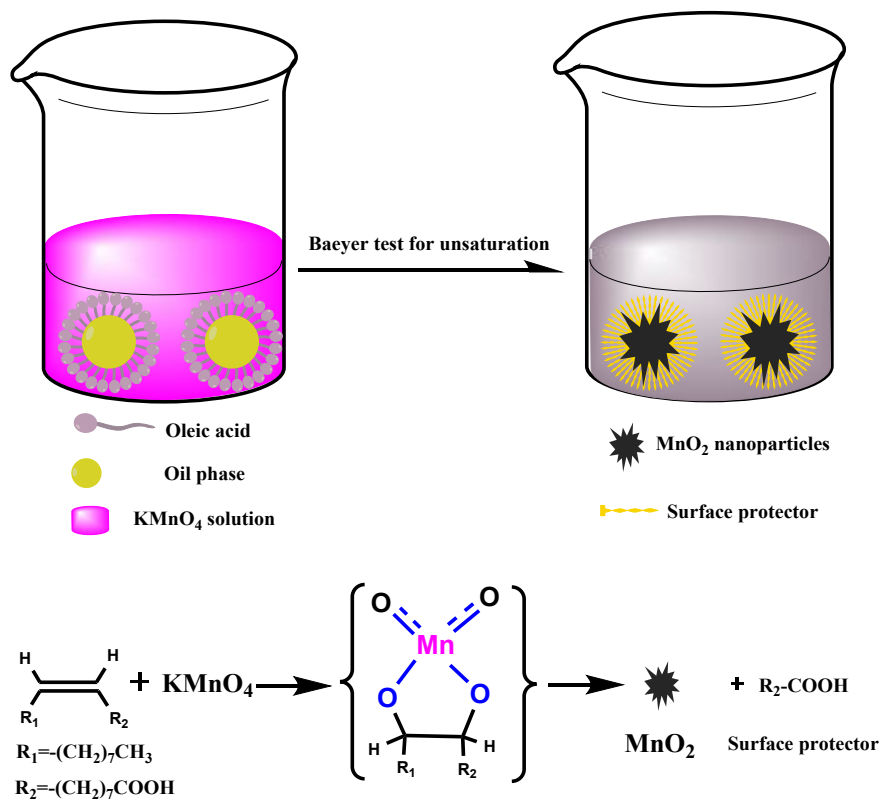

Scheme S1. Schematic illustration of $\mathrm{mMnO}_{2}$ nanoparticle synthesis. 


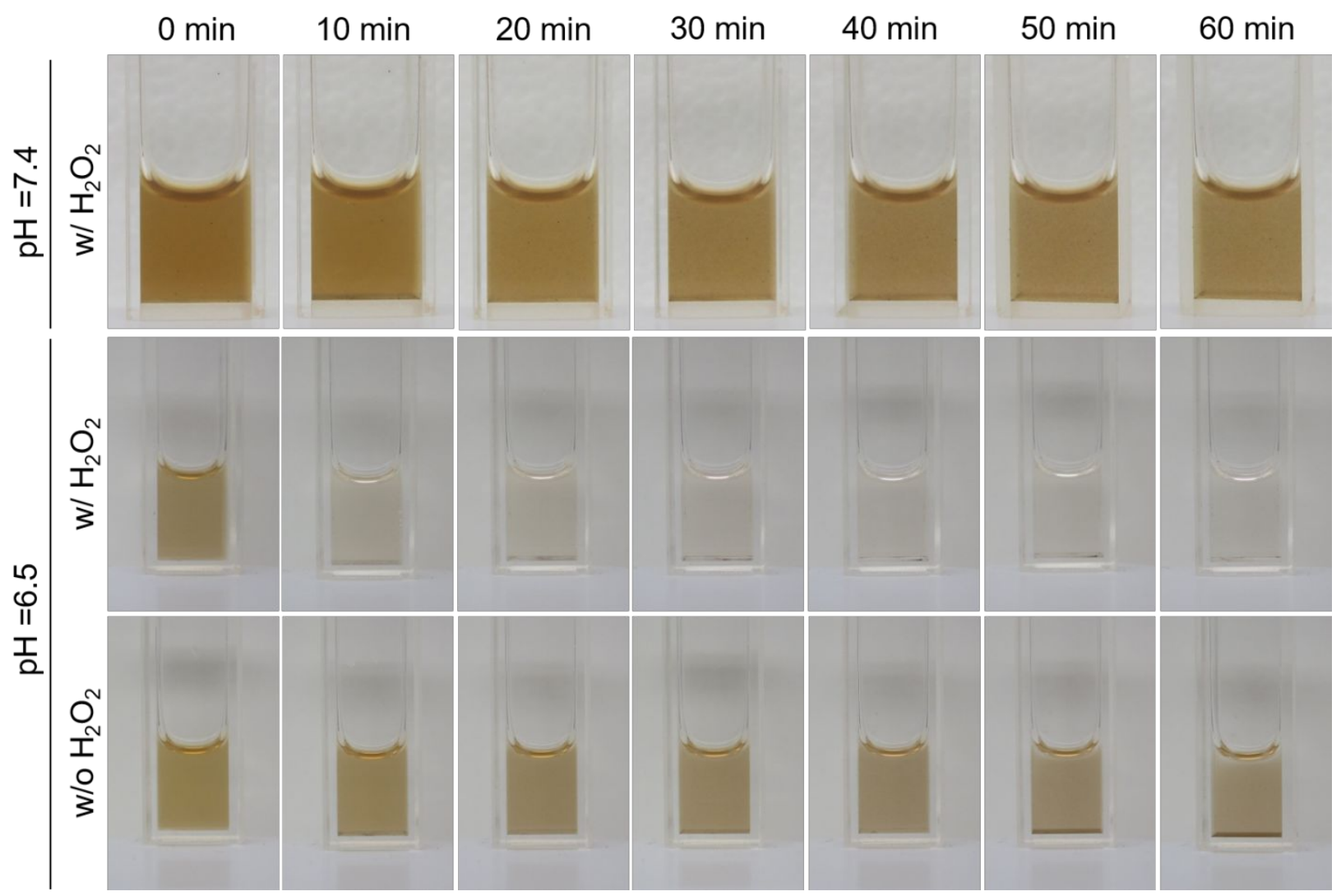

Figure S1. Temporal color changes upon mixing $\mathrm{mMnO}_{2}$ nanoparticles $(50 \mu \mathrm{g})$ with $\mathrm{H}_{2} \mathrm{O}_{2}(0.1 \mathrm{mM})$ in buffered solution at $\mathrm{pH} 7.4$ and 6.5 . 


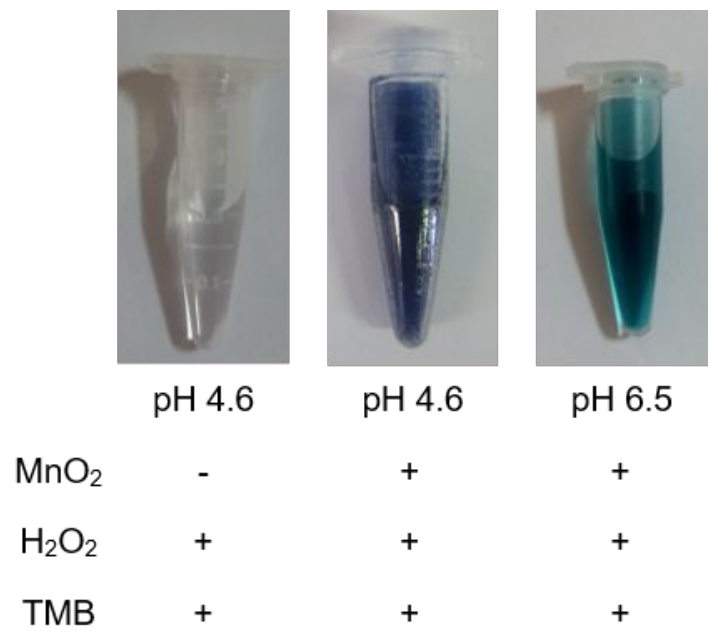

Figure S2. Peroxidase analysis with TMB.

$\mathrm{SCC}+\left[\mathrm{Ru}(\mathrm{dpp})_{3}\right] \mathrm{Cl}_{2}$

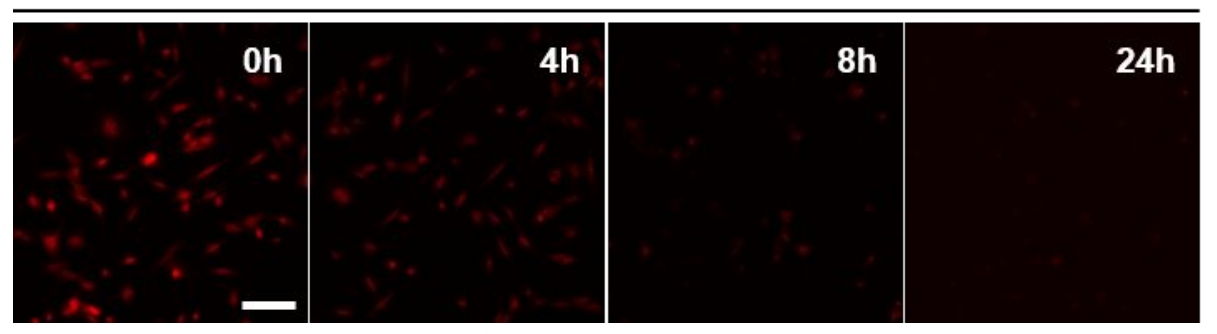

SCC

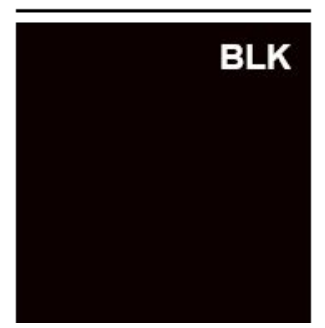

Figure S3. Fluorescence microscopy analysis of $\mathrm{mMnO}_{2}$ nanoparticle-catalyzed decomposition of intracellular $\mathrm{H}_{2} \mathrm{O}_{2}$ with $\mathrm{SCC}$ cells using $\left[\mathrm{Ru}(\mathrm{dpp})_{3}\right] \mathrm{Cl}_{2}$ as an oxygen probe. Scale, $100 \mu \mathrm{m}$. 

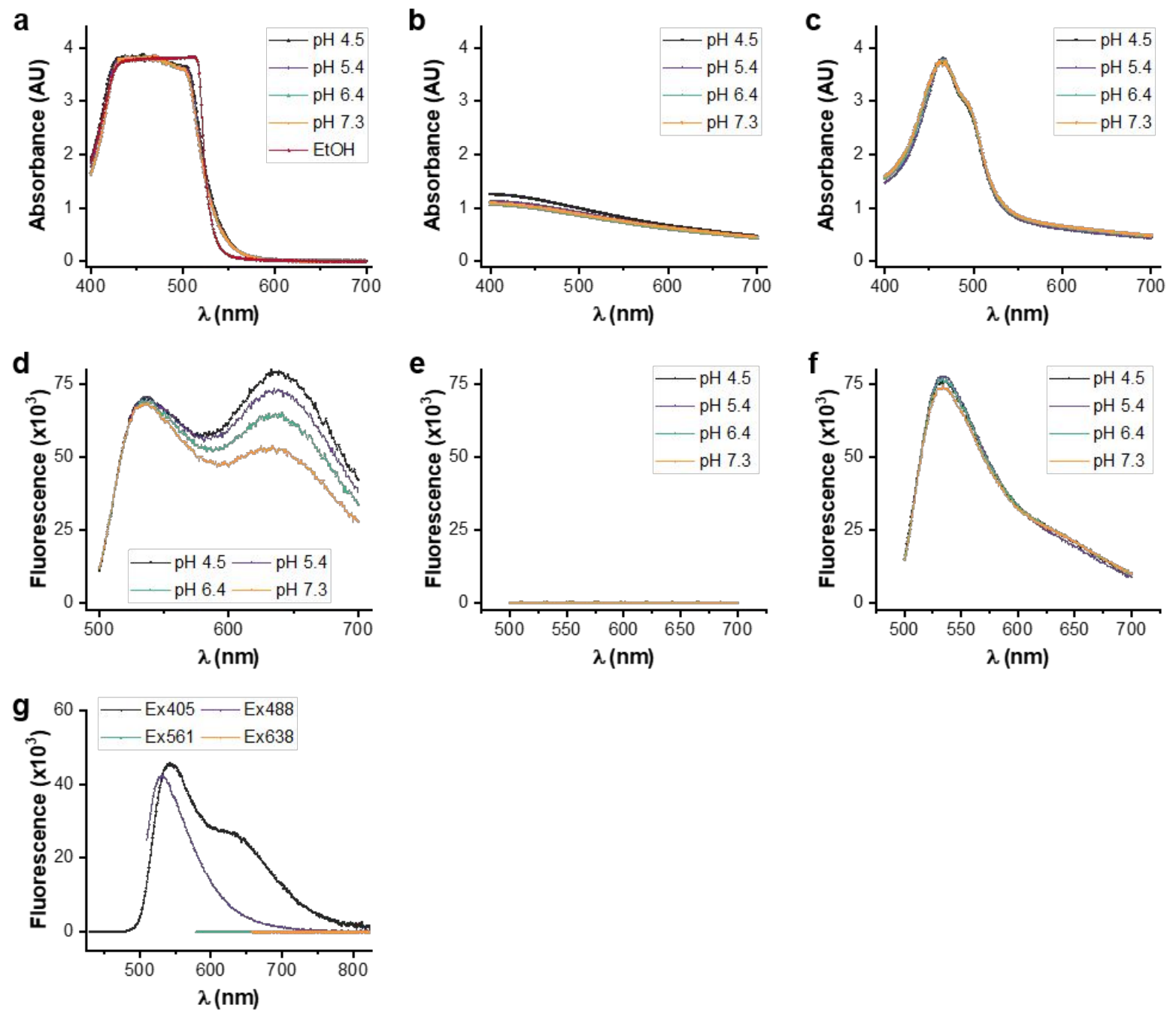

Figure S4. (a) Absorbance spectra of $\mathrm{AO}$ in $\mathrm{EtOH}$ and buffered solutions at different pHs. Absorbance spectra of $\mathrm{mMnO}_{2}$ (b) and $\mathrm{mMn}(\mathrm{AO})$ (c) nanoparticle dispersions in buffered solutions at different pHs. Fluorescence spectra with an excitation at $467 \mathrm{~nm}$ of $\mathrm{AO}$ solutions (d), $\mathrm{mMnO}_{2}(\mathbf{e})$, and $\mathrm{mMn}(\mathrm{AO})$ nanoparticle dispersion (f) at different pHs. (g) Fluorescence spectra of AO solution with an excitation at $405,488,561$, and $638 \mathrm{~nm}$. 


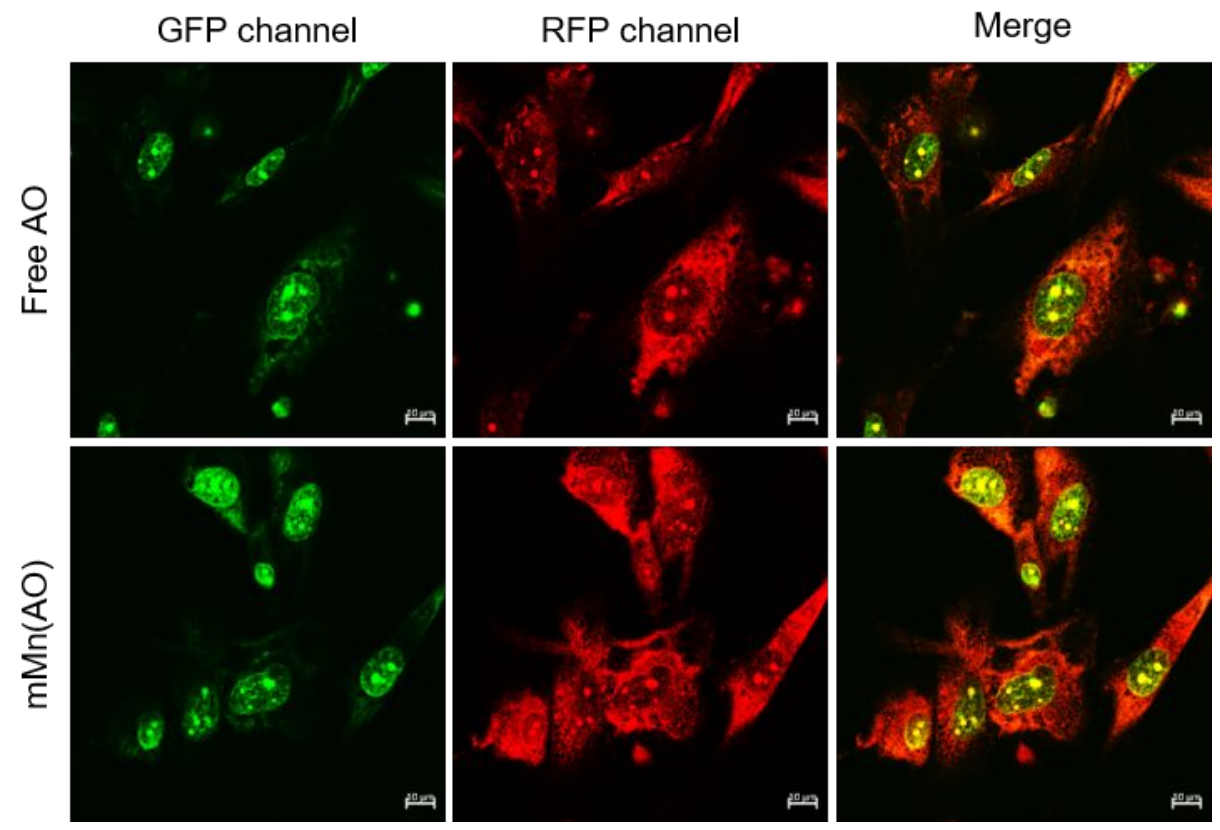

Figure S5. Fluorescence images of H1299 cells after incubating with free AO and mMn(AO) nanoparticles at $2.0 \mu \mathrm{g} / \mathrm{mL}$ of the $\mathrm{AO}$ equivalent. The monomer-form $\mathrm{AO}$ was visualized in GFP channel, and the aggregated-form AO was visualized in RFP channel. Scale bars, $10 \mu \mathrm{m}$. 

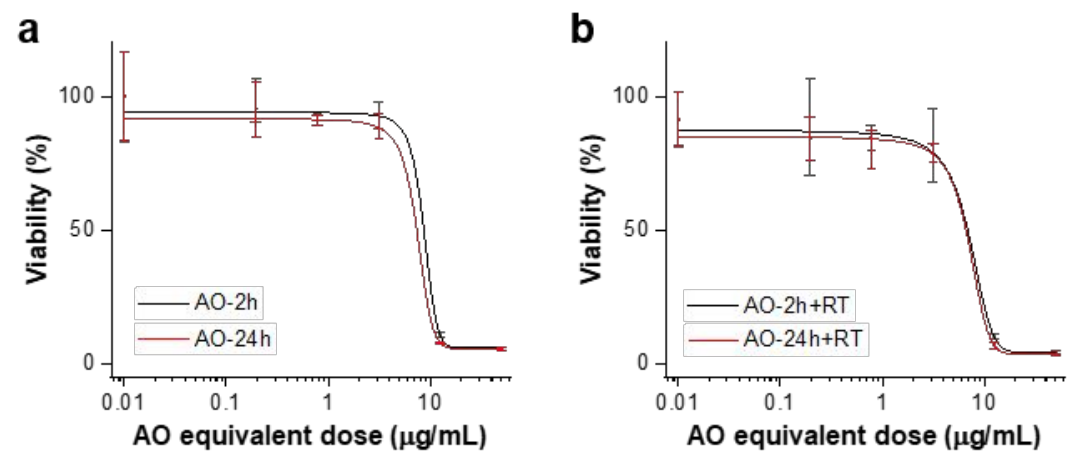

Figure S6. The MTT assay curves of free AO against H1299 cells with (a) and without (b) 5 Gy-radiation. AO-2h: cells were incubated with free AO for $2 \mathrm{~h}$ followed by medium change and further $24 \mathrm{~h}$-incubation. AO-24h: cells were incubated with free AO for $24 \mathrm{~h}$. Radiation was delivered right after the first $2 \mathrm{~h}-$ incubation. The results showed no significant difference between $\mathrm{AO}-2 \mathrm{~h}$ and $\mathrm{AO}-24 \mathrm{~h}$ in terms of the cytotoxicity. 


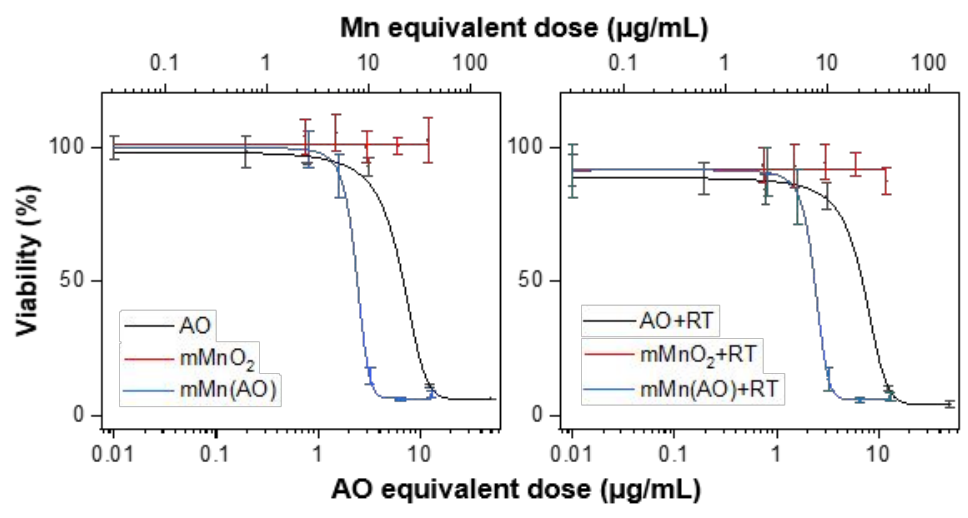

\begin{tabular}{ccccccc}
\hline & \multicolumn{2}{c}{$\mathrm{IC}_{20}(\mu \mathrm{g} \mathrm{AO} / \mathrm{mL})$} & \multicolumn{2}{c}{$\mathrm{IC}_{50}(\mu \mathrm{g} \mathrm{AO} / \mathrm{mL})$} & \multicolumn{2}{c}{$\mathrm{IC}_{80}(\mu \mathrm{g} \mathrm{AO} / \mathrm{mL})$} \\
& w/o RT & w/ RT & w/o RT & w/ RT & w/o RT & w/ RT \\
\hline $\mathrm{AO}$ & 4.06 & 3.29 & 6.87 & 6.78 & 10.16 & 10.00 \\
$\mathrm{mMn}(\mathrm{AO})$ & 1.92 & 1.70 & 2.44 & 2.36 & 3.03 & 2.98 \\
\hline
\end{tabular}

Figure S7. The MTT assay curves of free $\mathrm{AO}, \mathrm{mMnO}_{2}$ nanoparticles, and $\mathrm{mMn}(\mathrm{AO})$ nanoparticles against H1299 cells with and without 5-Gy radiation for $24 \mathrm{~h}$ of treatment and the $\mathrm{IC}_{20}, \mathrm{IC}_{50}$, and $\mathrm{IC}_{80}$ values of the corresponding treatments. 
Table S1

\begin{tabular}{ccccccc}
\hline \multicolumn{4}{c}{ Calculation based on 72h-MTT results } \\
\hline \multicolumn{4}{c}{ mMnAO } & \multicolumn{2}{c}{ AO } & \multicolumn{2}{c}{$\mathrm{mMnO}_{2}$} \\
\hline $\mathrm{A} 1$ & 8.4594 & 6.4307 & 21.9730 & 36.6649 & 71.7259 & 73.9022 \\
$\mathrm{~A} 2$ & 856.1745 & 1688.1636 & 633.0025 & 805.6607 & 304.9228 & 1667.0320 \\
$\mathrm{IgX}$ & -0.8086 & -0.2036 & -3.0366 & -0.1410 & -6.9516 & -1.5507 \\
$\mathrm{p}$ & -1.1340 & -6.1935 & -0.2748 & -7.7663 & -0.1235 & -1.2230 \\
$\mathrm{IC}$ & 0.10 & 0.01 & 0.53 & 0.02 & 4.66 & 0.42 \\
$\mathrm{IC}_{50}$ & 0.33 & 0.05 & 1.76 & 0.08 & $\mathrm{~N} / \mathrm{A}$ & N/A \\
$\mathrm{ER}_{20}$ & \multicolumn{2}{c}{8.21} & 31.67 & \multicolumn{2}{c}{10.99} \\
$\mathrm{ER}_{50}$ & 6.45 & 20.75 & \multicolumn{2}{c}{ N/A } \\
\hline
\end{tabular}

- Dose Response Eqt.: $y=A 1+(A 2-A 1) /\left(1+10^{\wedge}\left(\left(\lg X_{0}-x\right)^{*} p\right)\right)$

- Enhancement ratio (ER) at the corresponding inhibition concentration was calculated by using the following equation: $E_{x}=I C_{x}(w / o R T) / I C_{x}(w / R T)$

- The calculation was made alculation based on 72h-MTT results

Table S1. Calculation of radiation sensitization enhancement ratio (ER). $\mathrm{The}_{\mathrm{IC}}$ and $\mathrm{IC}_{50}$ values are based on $\mu \mathrm{g} / \mathrm{mL}$ equivalent $\mathrm{AO}$. 

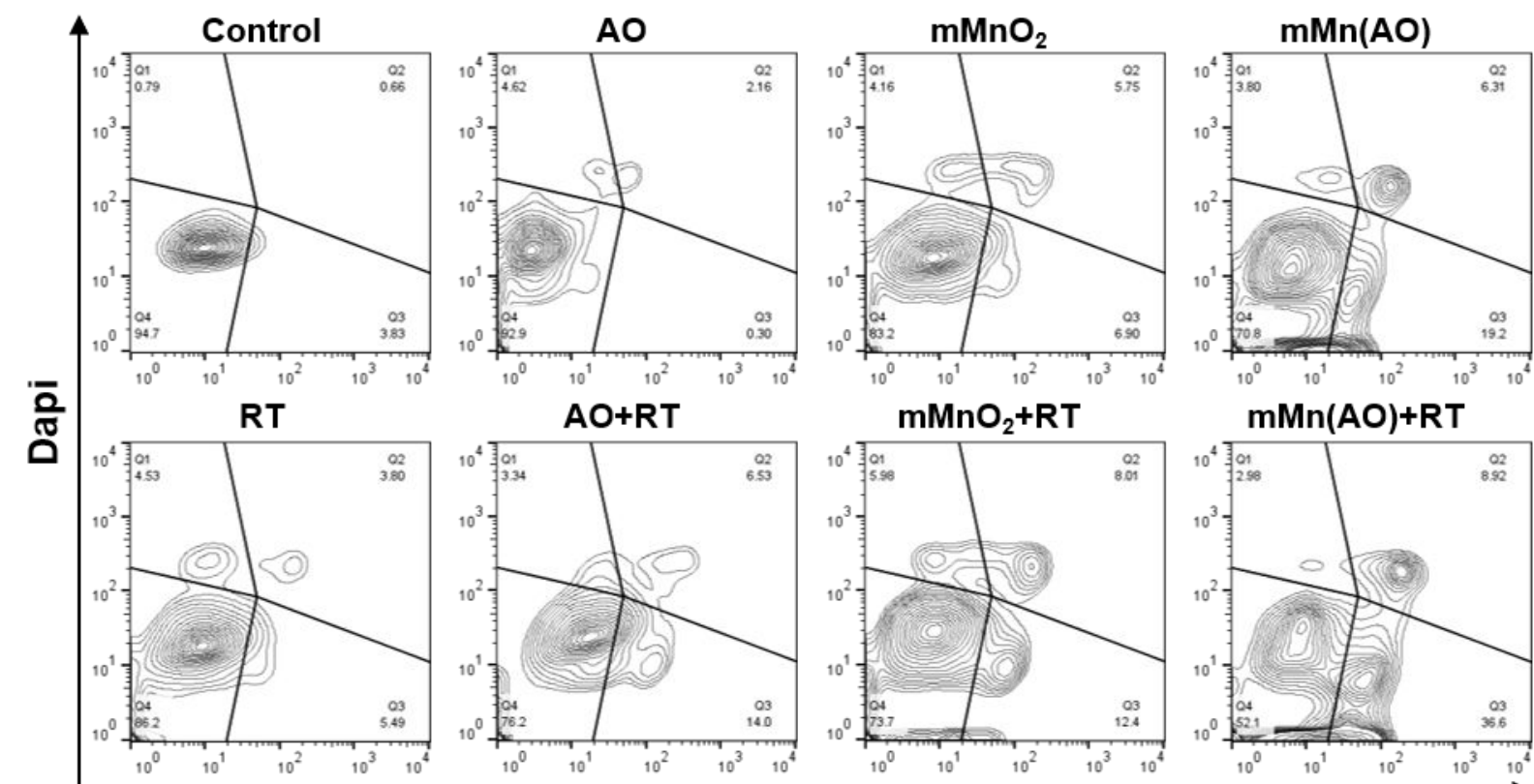

$\operatorname{mMn}(A O)+R T$

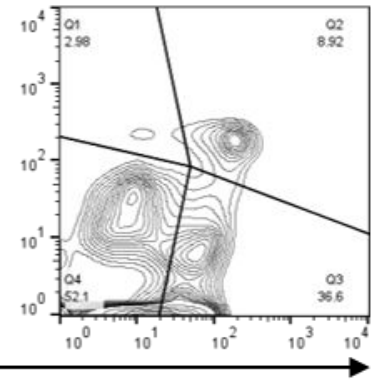

Annexin V-AF647

\begin{tabular}{lcccccccc}
\hline & \multicolumn{2}{c}{ Control } & \multicolumn{2}{c}{ AO } & \multicolumn{2}{c}{ mMnO $_{2}$} & \multicolumn{2}{c}{ mMn(AO) } \\
\hline & w/o RT & w/ RT & w/o RT & w/ RT & w/o RT & w/ RT & w/o RT & w/ RT \\
\hline Dapi+AV- $^{-0.79 \%}$ & $4.53 \%$ & $4.62 \%$ & $3.34 \%$ & $4.16 \%$ & $5.98 \%$ & $3.80 \%$ & $2.98 \%$ \\
Dapi+AV $^{+}$ & $0.66 \%$ & $3.80 \%$ & $2.16 \%$ & $6.53 \%$ & $5.75 \%$ & $8.01 \%$ & $6.31 \%$ & $8.92 \%$ \\
Dapi-AV $^{+}$ & $3.83 \%$ & $5.49 \%$ & $0.30 \%$ & $14.0 \%$ & $6.90 \%$ & $12.4 \%$ & $19.2 \%$ & $36.6 \%$ \\
Dapi-AV- & $94.7 \%$ & $86.2 \%$ & $92.9 \%$ & $76.2 \%$ & $83.2 \%$ & $73.7 \%$ & $70.8 \%$ & $52.1 \%$ \\
\hline
\end{tabular}

Figure S8. Flow cytometry analysis with DAPI and Annexin V double stained H1299 cells. The cells were pre-treated with $\mathrm{AO}, \mathrm{mMnO}_{2}$, or $\mathrm{mMn}(\mathrm{AO})(1.0 \mu \mathrm{g} \mathrm{AO} / \mathrm{mL})$ in the presence or absence of RT (5 Gy). 

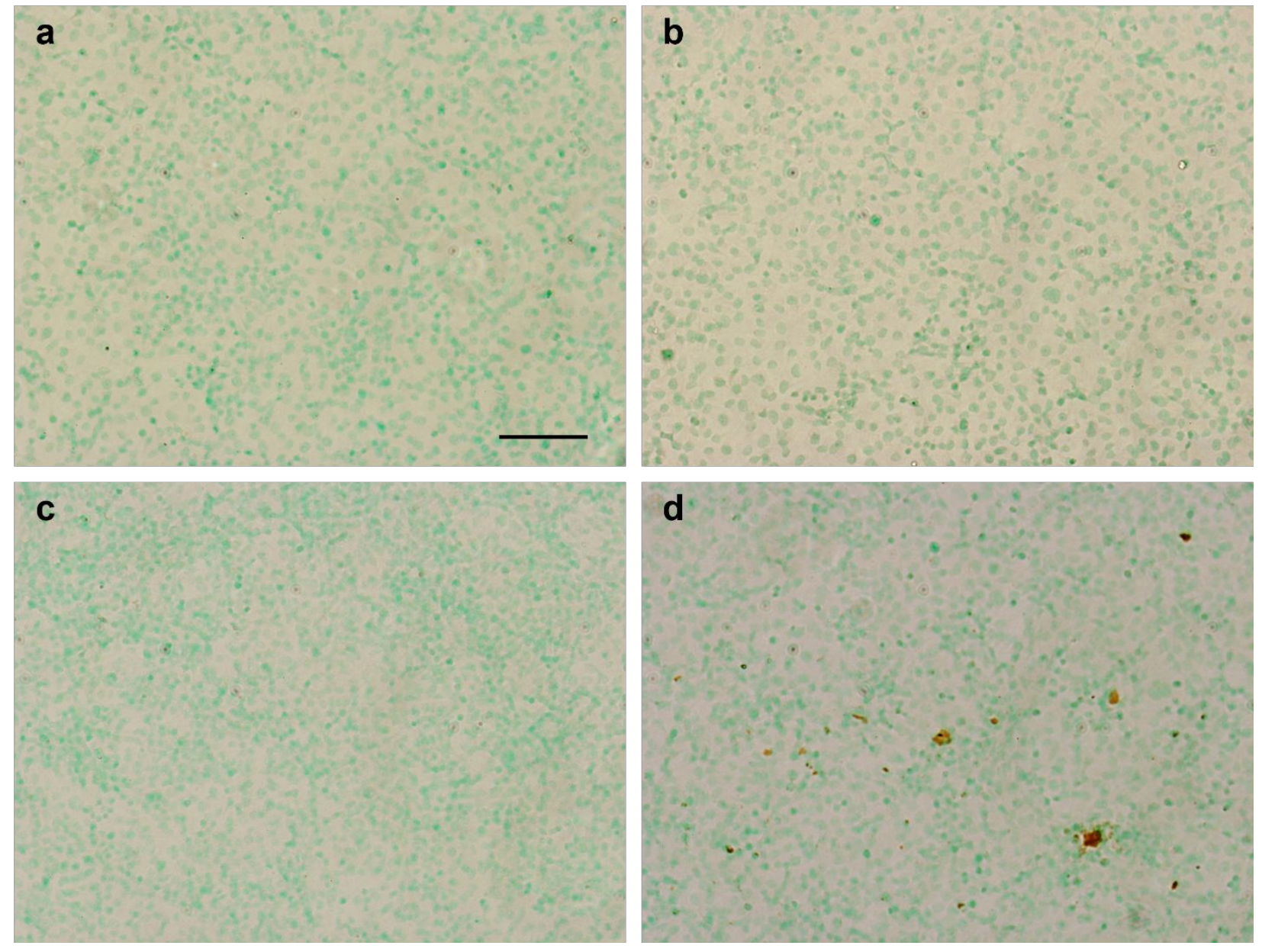

Figure S9. In situ apoptosis staining images of different treatments against H1299 cells. (a) control; (b) $\mathrm{AO} ;(\mathbf{c}) \mathrm{mMnO}_{2}$ nanoparticles; (d) $\mathrm{mMn}(\mathrm{AO})$ nanoparticles. All treatments were done at a dose equivalent to $1.0 \mu \mathrm{g} / \mathrm{mL}$ of AO. The cytoplasm area was stained into green color with methylene green. The nuclei of apoptotic cells were stained into dark brown dots with HRP-DAB. Scale bar, $100 \mu \mathrm{m}$. 

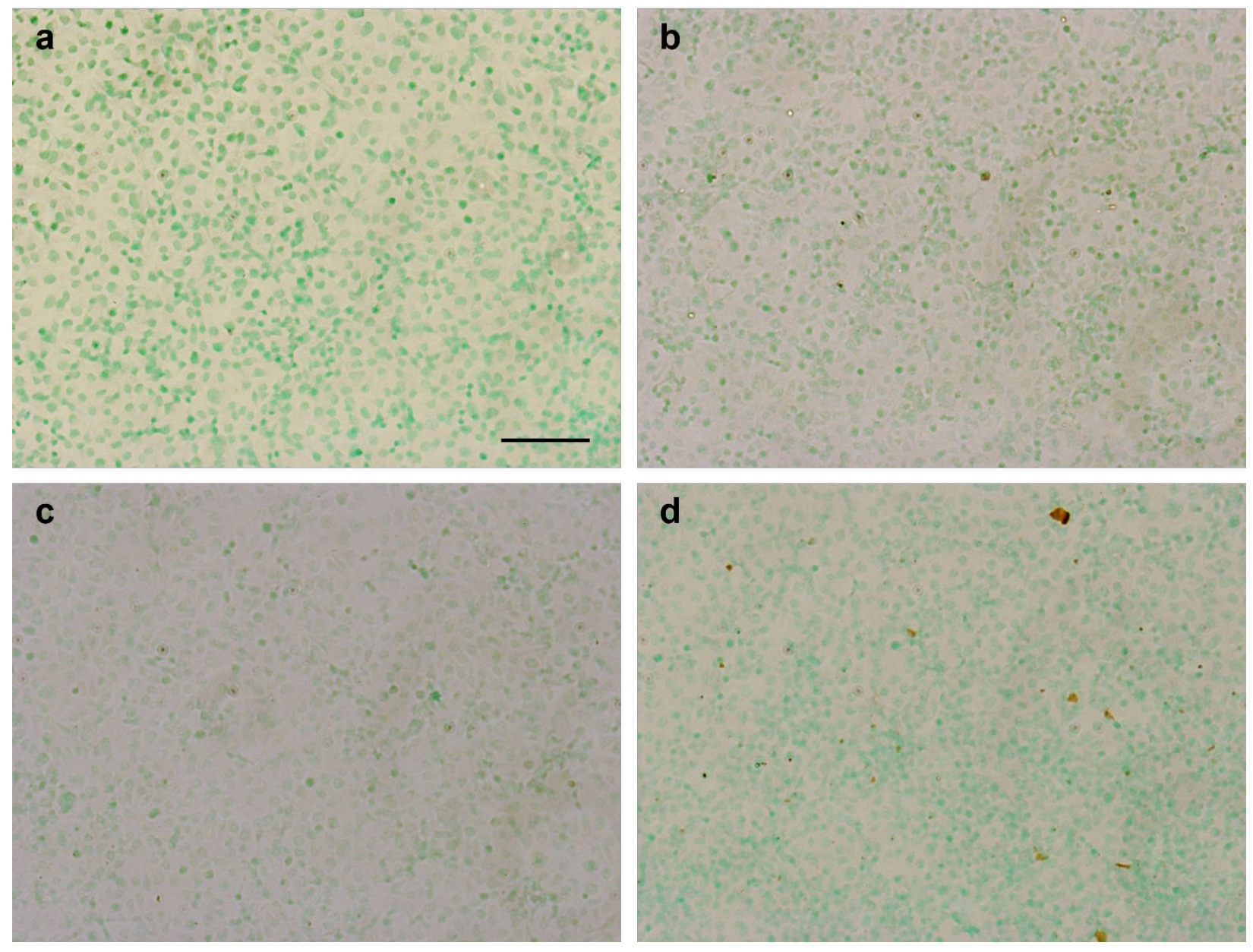

Figure S10. In situ apoptosis staining images of different treatments against H1299 cells. (a) RT; (b) $\mathrm{AO}+\mathrm{RT}$; (c) $\mathrm{mMnO}_{2}+\mathrm{RT}$; (d) $\mathrm{mMn}(\mathrm{AO})+\mathrm{RT}$. 5 Gy was the radiation dose, and all treatments were conducted at a $\mathrm{AO}$ equivalent dose at $1.0 \mu \mathrm{g} / \mathrm{mL}$. The cytoplasm area was stained into green color with methylene green. The nuclei of apoptotic cells were stained into dark brown dots with HRP-DAB. Scale bar, $100 \mu \mathrm{m}$. 


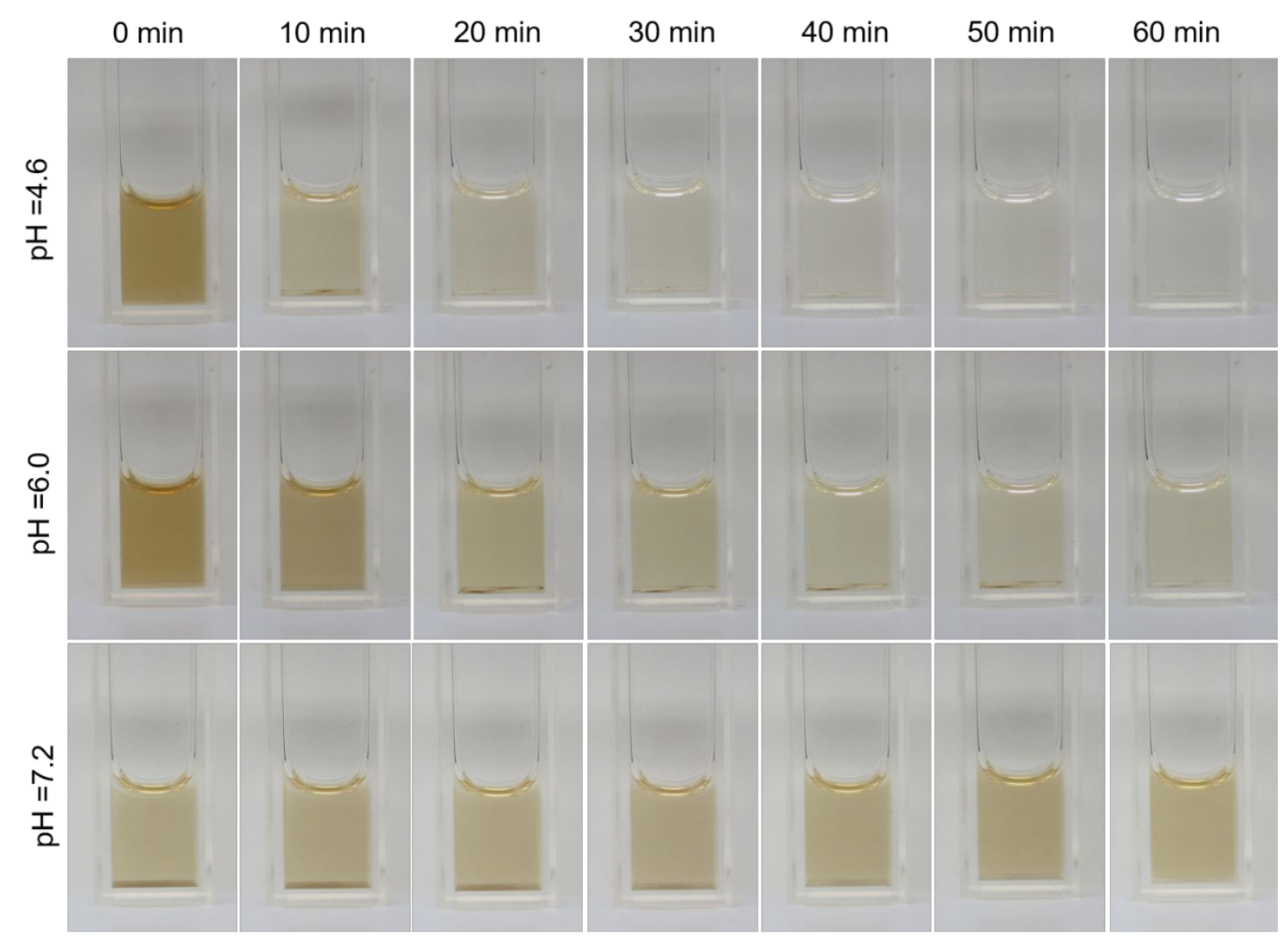

Figure S11. Temporal decomposition of $\mathrm{mMnO}_{2}$ nanoparticles at different $\mathrm{pHs}$. 

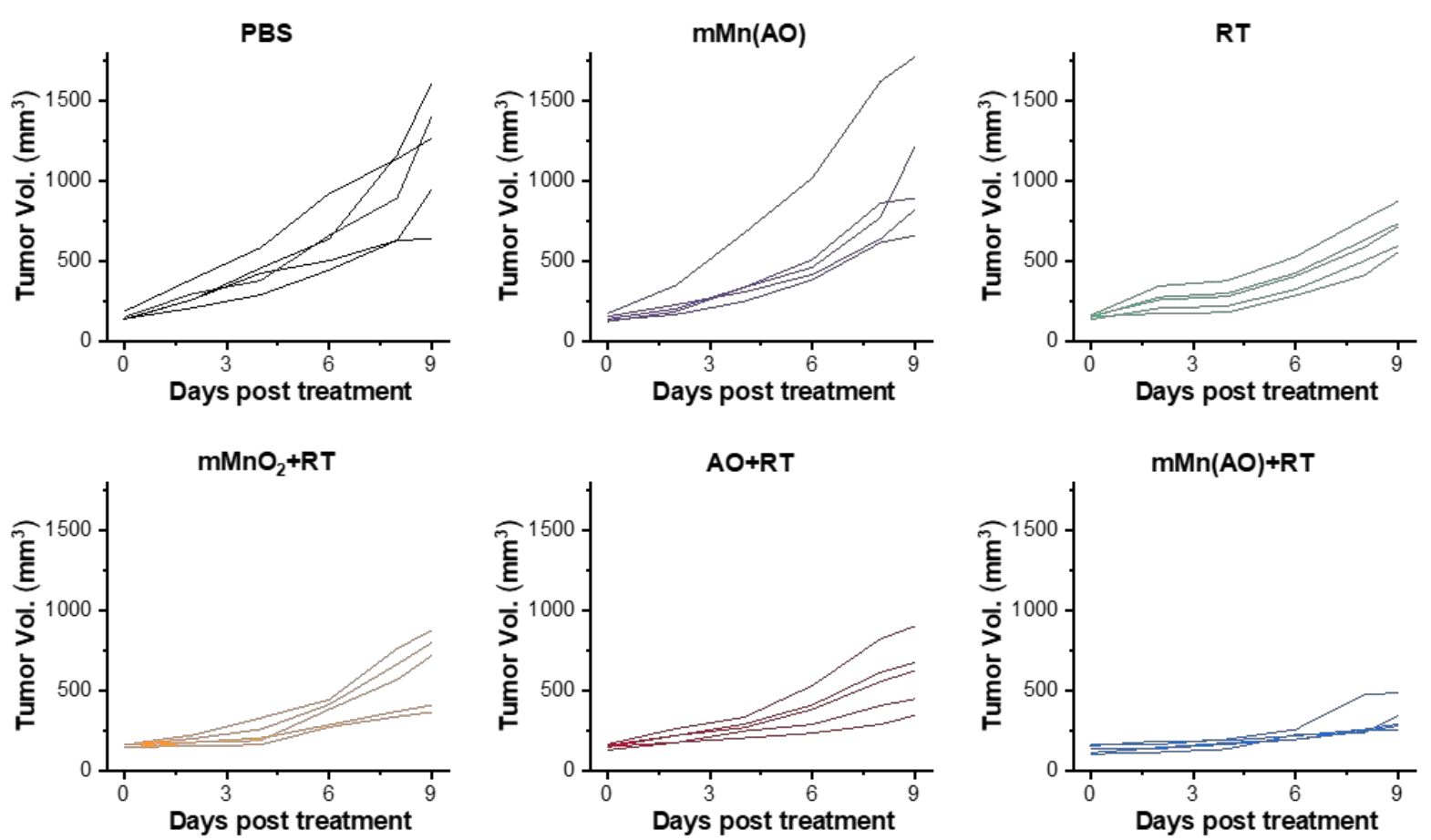

Figure S12. Tumor growth curves of each individual mouse post treatments.
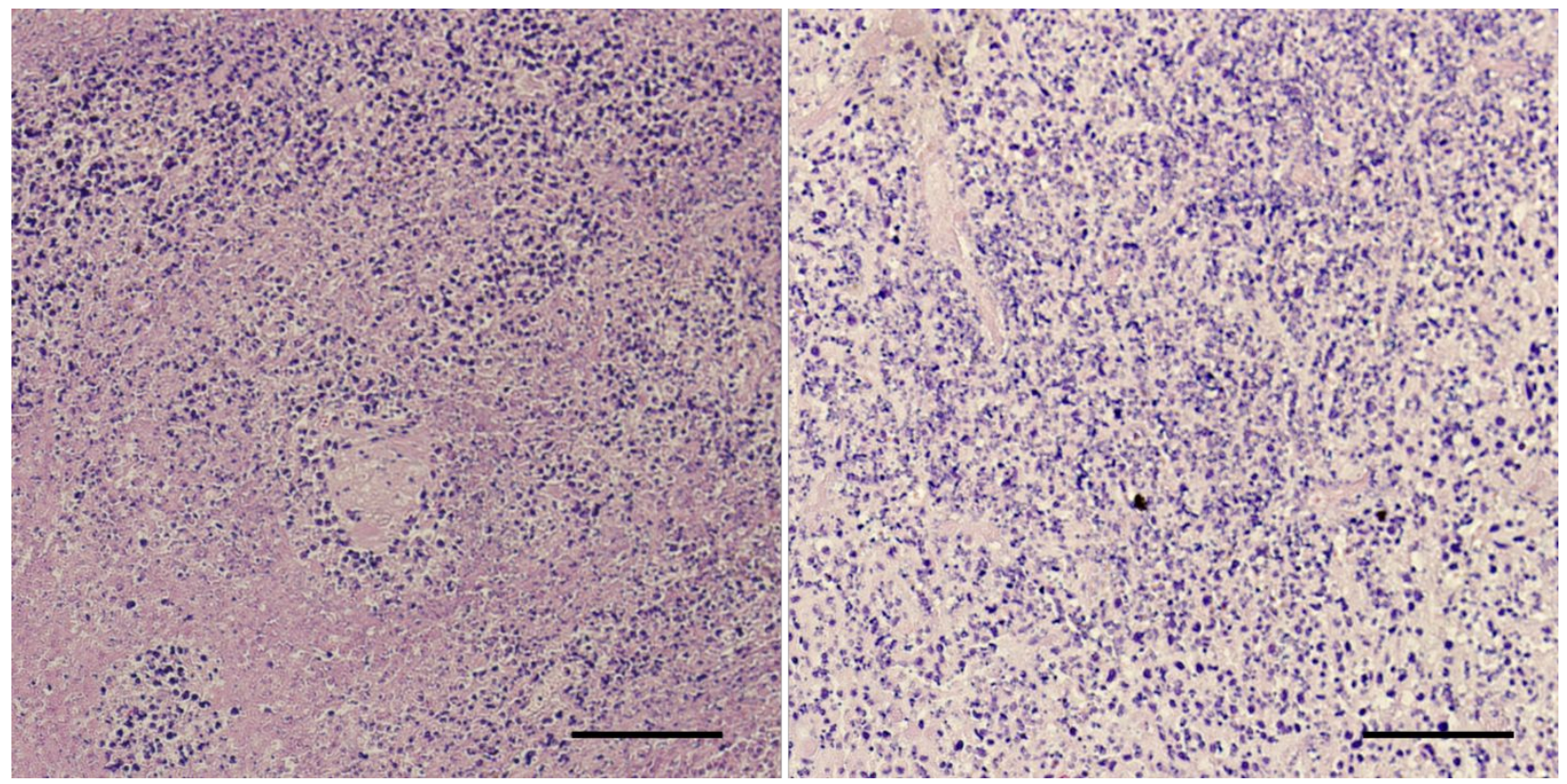

Figure S13. H\&E staining of mMn(AO)+RT tumor tissues collected on Day 9. Scale bar, $100 \mu \mathrm{m}$. 